\author{
Military Technical College \\ Kobry El-kobbah, \\ Cairo, Egypt
}

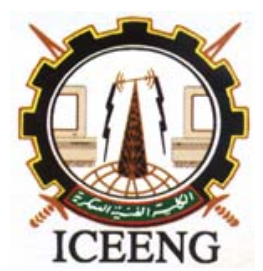

\title{
THERMAL IMAGING RADIOMETER OVERALL PERFORMNCE PARAMETERS AND ITS PROBABILITY OF TARGET DISCRIMINATION
}

\author{
H.ELSHEIKH* , I.ABD-ELDAYEM*, S.MOWAD**
}

\begin{abstract}
The minimum resolvable temperature difference (MRTD) is the overall performance parameter of any thermal imaging radiometer (TIR). MRTD combine the sensitivity and resolution of the TIR. It accurately describes the interrelationship for a particular TIR. MRTD is used especially tactically oriented (fire control and targeting) organizations, as the primary performance measure and is used with a target and atmospheric parameters to give a probability of target discrimination. This paper is directed toward constructing a software program for the calculation and plotting the MRTD and a probability of target discrimination. These parameters can be used as a tutorial or a procedure to determine how well TIR parameters perform in overall imaging system scenario for particular targets, backgrounds, atmospheric conditions allowing a quick evaluation of the TIR in different environments.
\end{abstract}

\section{KEY WORD}

Thermal imaging radiometer, sensitivity, Resolution, Minimum resolvable temperature difference and Probability of object discrimination.

\section{NOMENCLATURE}

$\begin{array}{ll}\rho_{o} & \text { The spatial frequency } \\ \Delta T_{t g t} & \text { The target-background differential temperature } \\ \Delta T_{a p p} & \text { The target-background apparent differential temperature } \\ \sigma & \text { The value of slit width angle for which the impulse response } \\ & \text { function of the TIR is equal to the fraction 1/e of its maximum } \\ & \text { value. } \\ \sigma^{\prime} & \text { the extinction coefficient } \\ \Delta v & \text { The electrical bass band } \\ D^{*}(\lambda) & \text { The specific detectivity } \\ \tau_{o p}(\lambda) & \text { The spectral transmission of the optical system } \\ \tau_{a t m} & \text { The atmospheric transmission } \\ A & \text { The detector area } \\ * \ldots \text { ARMED FORCES } & \end{array}$




$\begin{array}{ll}d_{c} & \text { The critical dimension of the target } \\ \text { FLIR } & \text { The forward-looking infrared } \\ \text { FOV } & \text { The field of view } \\ h & \text { The height of the target. } \\ \text { IFOV } & \text { The instantaneous field of view } \\ \text { MRTD } & \text { The Minimum resolvable temperature difference } \\ N^{\prime} & \text { The numerical aperture } \\ N & \text { The number of cycles across the critical target dimension } \\ N_{50} & \text { The corresponding } 50 \% \text { cycle criteria } \\ R & \text { The range from the TIR to the target } \\ \text { TIR } & \text { The thermal imaging radiometer } \\ T T P F & \text { The target transfer probability function } \\ W & \text { The width of the target. }\end{array}$

\section{INTRODUCTION}

The radiometer systems that utilizing thermal imaging techniques have been developed significantly within the past decades. These developments represent a very vital threats. It is necessary to know the abilities of these thermal imaging radiometers (TIRs) to detect and discriminate the weapon radiometers and the ground installations. The thermal imaging detection technique is strongly dependent on several factors, such as: the characteristics of TIRs, the nature of the target, and the atmospheric conditions which affect too much the propagation of the infrared radiation. From another point of view it is required to choose correctly the proposed TIRs in the exiting conditions. This choice will affect on the target discrimination probabilities. One of the primary system level tactical TIRs performance is the probability of performing a visual discrimination task (detection, recognition, and identification) where the TIR is at a given range from a target. The detection task is the process of determining if a target is present. The recognition task is the gross classification of the target into imaging groups such as trucks, cars and the like. The identification task is a higher level of discrimination within major group. Here, we focus on static performance, which refers to the ability of the observer to perform a discrimination task while having prior knowledge that a target is within the FOV and given an infinite amount of time. The target, scene, and TIR are stationary.

The performance measures of discrimination were first quantified by Johnson [8] in 1958 and have been modified slightly over the years for infrared sensors since the work was based on image intensifier imagery. As a result of Johnson's original performance measures, the current discrimination criteria (with $50 \%$ probabilities) [4] are shown in Table 1. The initial discrimination criteria developed by Johnson were modified to be relevant for, the forward-looking infrared (FLIR) performance prediction by Ratches, et al. [9] in 1975. This performance prediction as a function of range was based on a one-dimensional model using only the horizontal MRTD. The work has been further modified to reflect the two-dimensional model that is derived using both the horizontal and vertical MRTDs. The $50 \%$ probability cycle criteria for the one- and two-dimensional MRTDs are shown in table 1. In this paper, we use the inframetrics 760 [2] and FLIR92 [5] TIRs operating in the spectral infrared band (8-12 $\mu \mathrm{m})$. 
Table (1) Discrimination criteria

\begin{tabular}{|c|c|c|c|}
\hline $\begin{array}{c}\text { Discrimination } \\
\text { Level }\end{array}$ & Meaning & $\begin{array}{l}\text { One-dimensional } \\
\text { cycles across } \\
\text { minimum } \\
\text { dimension }\end{array}$ & $\begin{array}{l}\text { Two-dimensional } \\
\text { cycles across critical } \\
\text { dimension, } N_{50}\end{array}$ \\
\hline Detection & $\begin{array}{c}\text { An object is } \\
\text { Present }\end{array}$ & 1 & 0.75 \\
\hline Recognition & $\begin{array}{c}\text { Class to which an } \\
\text { object belongs } \\
\text { (e.g., human, tank, } \\
\text { truck) }\end{array}$ & 4.0 & 3.0 \\
\hline Identification & $\begin{array}{c}\text { Object is } \\
\text { discerned with } \\
\text { enough clarity to } \\
\text { specify type } \\
\text { (M1A1, T-62, } \\
\text { T-72 Tank) }\end{array}$ & 8.0 & 6.0 \\
\hline
\end{tabular}

\section{MINIMUM RESOLVABLE TEMPERATURE DIFFERENCE MATHIMATICAL MODEL}

MRTD is a design criterion that flows down from computer-aided performance models. The detection and recognition ranges can be predicted from an MRTD curve for a given target size, target temperature, background temperature and a given atmospheric attenuation. MRTD can be calculated $[1,3]$ using the following formula:

$$
M R T D=\frac{\frac{4 N^{\prime 2} \sqrt{A \Delta v}}{\sqrt{A} \tau_{O P}}\left[\int_{\lambda_{a}}^{\lambda_{b}} \frac{d}{d T} \frac{d R}{d \lambda} D^{*}(\lambda) d \lambda\right]^{-1}}{\exp \left[-\pi^{2} \sigma^{2} \rho_{o}^{2}\right]}
$$

The MRTD curve will have a vertical asymptote at the cutoff spatial frequency. Because all thermal-imager systems operate with a finite detector size, this asymptote frequency cannot be greater than the inverse of the detector angular subtense.

\section{DETERMINATION OF THE PROBABILITIES OF OBJECT DISCRIMINATIONS}


The procedure for producing the probability of detection, recognition, or identification curve is as follows. Consider the process flow given in Fig.1.

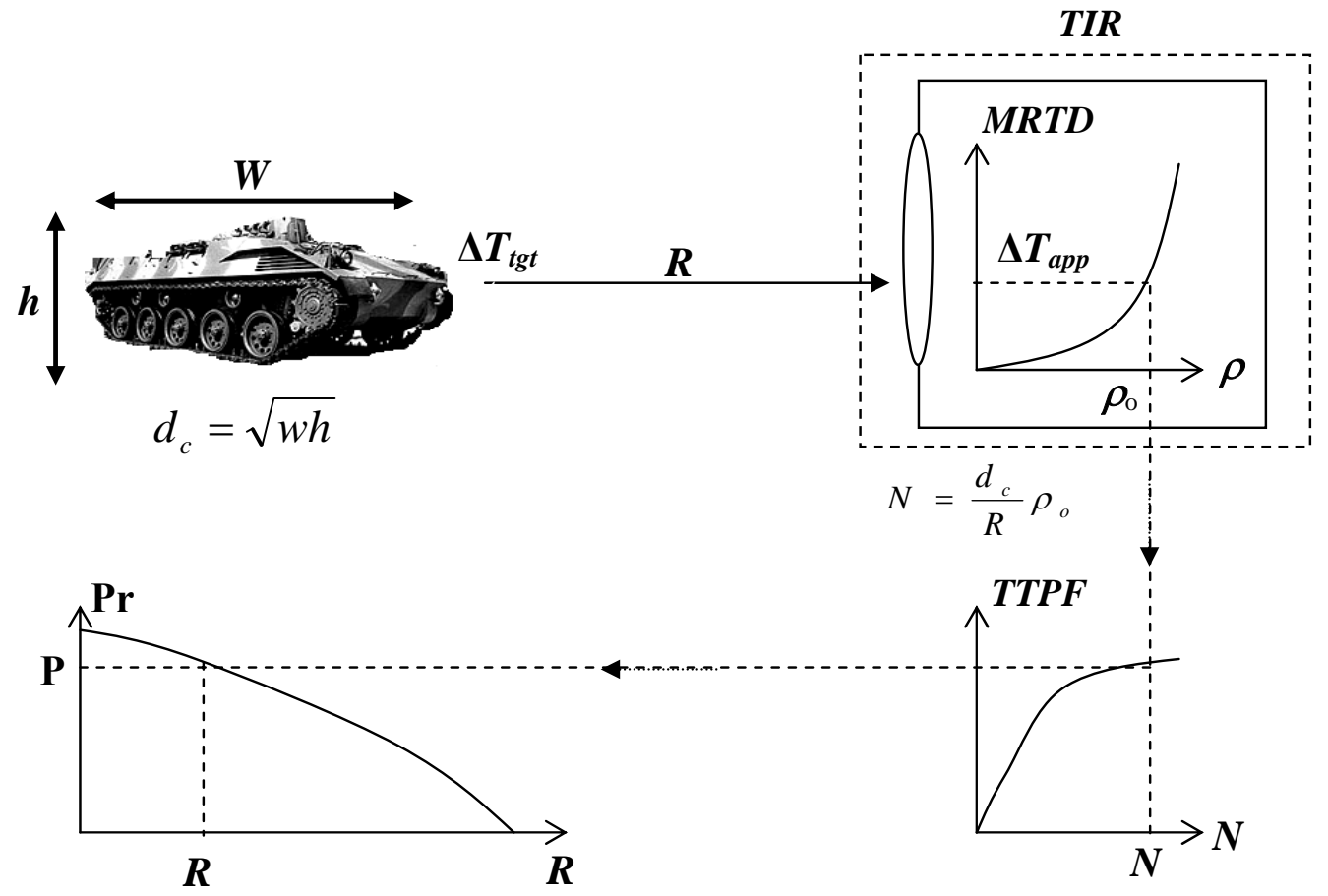

Fig. (1) Probability of discrimination process.

There are four parameters that are needed to generate a static probability of discrimination curve as a function of range. They are estimated target-to-background temperature differential, an estimated height and width of a target, an estimate for the atmospheric transmission of the band of interest for a number of ranges around the ranges of interest, and the TIR one or two-dimensional MRTD.

The target parameters are determined first. The critical dimension of the target $d_{c}$ is taken as the geometric mean of the target height $(h)$ and width $(W)[4]$ as:

$$
d_{c}=\sqrt{w h}
$$

The target-to-background temperature difference is then estimated based on target and background characteristics. For ground targets, these differential temperatures are usually between 1.25 and $4.0 \mathrm{deg} \mathrm{C}$. The apparent differential temperature is then determined. There are numerous techniques for determining the apparent differential temperature [4].

Once an apparent differential temperature is obtained, the highest corresponding spatial frequency that can be resolved by the TIR is determined. This is accomplished by finding the spatial frequency $\rho_{o}$ that matches the target apparent differential temperature on the MRTD curve. The number of cycles across the critical target dimension that can actually be resolved by the TIR [4] is 


$$
N=\rho_{o} \frac{d_{c}}{R}
$$

The probability of discrimination is determined using the target transfer probability function (TTPF). The level of discrimination (detection, recognition, or identification) is selected from Table 2 and the corresponding $50 \%$ cycle criteria, $\mathrm{N}_{50}$, is taken from the table 2. The probability of detection, recognition, or identification is then determined with the TTPF [4] as

$$
\text { TTPF }=\frac{\left(\frac{N}{N_{50}}\right)^{2.7+0.7\left(\frac{N}{N_{50}}\right)}}{1+\left(\frac{N}{N_{50}}\right)^{2.7+0.7\left(\frac{N}{N_{50}}\right)}}
$$

The function is shown in Fig.1. The probability of discrimination task is then assigned to the particular range. A typical probability of discrimination curve has the probability plotted as a function of range. Therefore, the above procedure would be repeated for a number of different ranges. This procedure provides the conversion of the TIR performance MRTD specification to an operation performance specification. In fact, it is used frequently to give the probability of discrimination regardless of the TIR MRTD source. We used the Inframetrics 760 and FLIR92 MRTDs shown in Fig.2.

It is desired to determine the TIR probability functions of detection, recognition and identification of a standard NATO target at a range up to $20 \mathrm{Km}$. A standard NATO target, which is representative of a front aspect view of a tank, has a width of $2.3 \mathrm{~m}$, a height of $2.3 \mathrm{~m}$, and a target-to-background differential temperature of $1.25 \mathrm{deg} \mathrm{C}$. The conditions are clear, the standard U.S. atmosphere, and altitude of $0.5 \mathrm{~km}$ above sea level. And the extinction coefficient is 0.1054 per kilometer. the atmosphere transmittance over ranges from $1 \mathrm{~km}$ to $20 \mathrm{~km}$ is determined [4] using the following relation

$$
\tau_{\text {atm }}(R)=e^{-\sigma^{\prime} \times R}
$$

The calculation begins by determining an apparent target-to-background differential temperature as seen by the TIR. In the long wave, it is common to multiply the actual differential temperature by the atmospheric transmission to determine the apparent differential temperature [4] using the following relation

$$
\Delta T_{\text {app }}(R)=\Delta T_{\text {tgt }} \tau_{a t m}(R)
$$


For each $\Delta T_{\text {app }}(R)$, a frequency $\rho_{o}$ must be determined using both Inframetrics 760 and FLIR92 MRTDs as shown in Fig.1. Recall that $\rho_{o}$ is the highest (critical) spatial frequency that can be resolved by the TIR at a given $\Delta T_{a p p}(R)$ as a function of range.

The critical numbers of cycles are then calculated using the critical frequency, the range, and the critical target dimension using equation (3). The $50 \%$ number of cycles across target $\mathrm{N}_{50}$ for detection, recognition, and identification were taken as $0.75,3.0$, and 6.0, respectively for two dimensional FLIR92 MRTD and the 50\% number of cycles across target $\mathrm{N}_{50}$ for detection, recognition, and identification were taken as taken as 1.0, 4.0, and 8.0, respectively for one dimensional Inframetrics 760 MRTD as in Table 1. Finally, We calculate the probabilities of identification, recognition, and detection using equation (4).

It is convenient to represent the acquisition process with the MRTD and the target "load line" on the same graph. The MRTD curve can be scaled for a detection, recognition an identification ranges [4]. The system MRTD curve is re-plotted with a detection, recognition and identification ranges using

$$
\rho_{o}=\frac{N_{50}}{d_{C}} R
$$

The apparent differential temperature of the target can be plotted as a function of range. Because it takes an $\mathrm{N}_{50}$ of across the target to obtain a 50 percent probability of detection, recognition and identification. The two lines cross at a point where the system provides the detection, recognition and identification ranges for a $50 \%$ probability of discrimination $[6,7]$.

\section{MODELING OF THERMAL IMAGING RADIOMETER OVERALL PERFORMNCE PARAMETER AND ITS PROBABILITY OF OBJECT DISCRIMINATION}

A software program is developed using a computer programming language, MATLAB package, for modeling the MRTD and their probability of object discriminations. The idea of the soft ware program is to calculate and plot MRTD and the probability of object detection, recognition and identification and also the detection, recognition and identification ranges as follows:

First, we perform a mathematical model for MRTD as illustrated by formula (1).

Second, the probability of a standard NATO target discriminations and their ranges of detection, recognition and detection are computed for both Inframetrics 760 and FLIR92 TIR.

\section{RESULTS AND DISCUSSION}

Fig.2 illustrate the Inframetrics 760 which has a cut off spatial frequency $0.55 \mathrm{c} / \mathrm{mrad}$. and the FLIR92 MRTD which has a cut off spatial frequency equal to $3.8 \mathrm{c} / \mathrm{mrad}$. The FLIR92 MRTD is better than the Inframetrics 760 MRTD because it has lower MRTD values at higher spatial frequencies. 

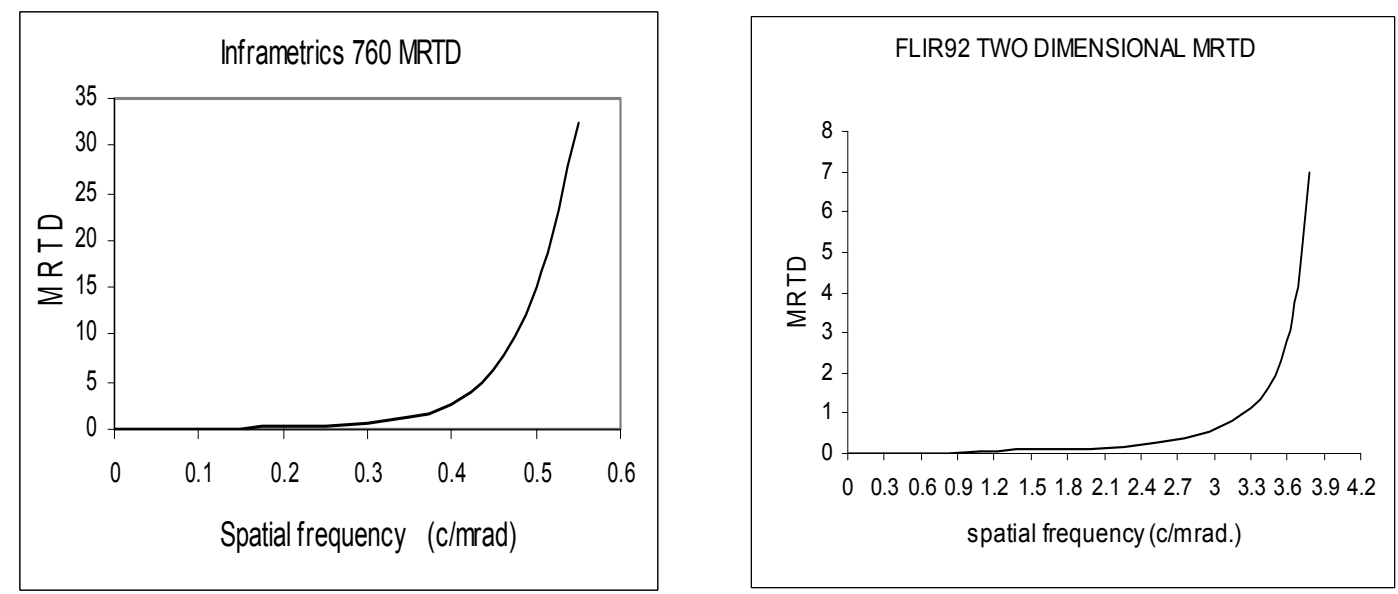

Fig.2 Inframetrics 760 and FLIR92 MRTDs curves

Fig.3 illustrate the graphical representation of the 50\% probabilities of identification, recognition and detection of the Inframetrics 760 and FLIR92. The 50\% number of cycles across target $\mathrm{N}_{50}$ for detection, recognition, and identification were taken as taken as 1.0, 4.0, and 8.0, respectively for one dimensional Inframetrics 760 MRTD and the $50 \%$ number of cycles across target $\mathrm{N}_{50}$ for detection, recognition, and identification were taken as $0.75,3.0$, and 6.0 , respectively for two dimensional FLIR92 MRTD. At a target range of $4 \mathrm{~km}$ from the TIRs, the Inframetrics 760 probability of detection, recognition and identification is equal to $0.0078,0.0002$ and 0.0001 respectively and the FLIR92 probability of detection, recognition and identification is equal to $0.98,0.17$ and 0.03 respectively. Then the FLIR92 probability of detection, recognition is better than the Inframetrics 760 probability of detection, recognition and identification.
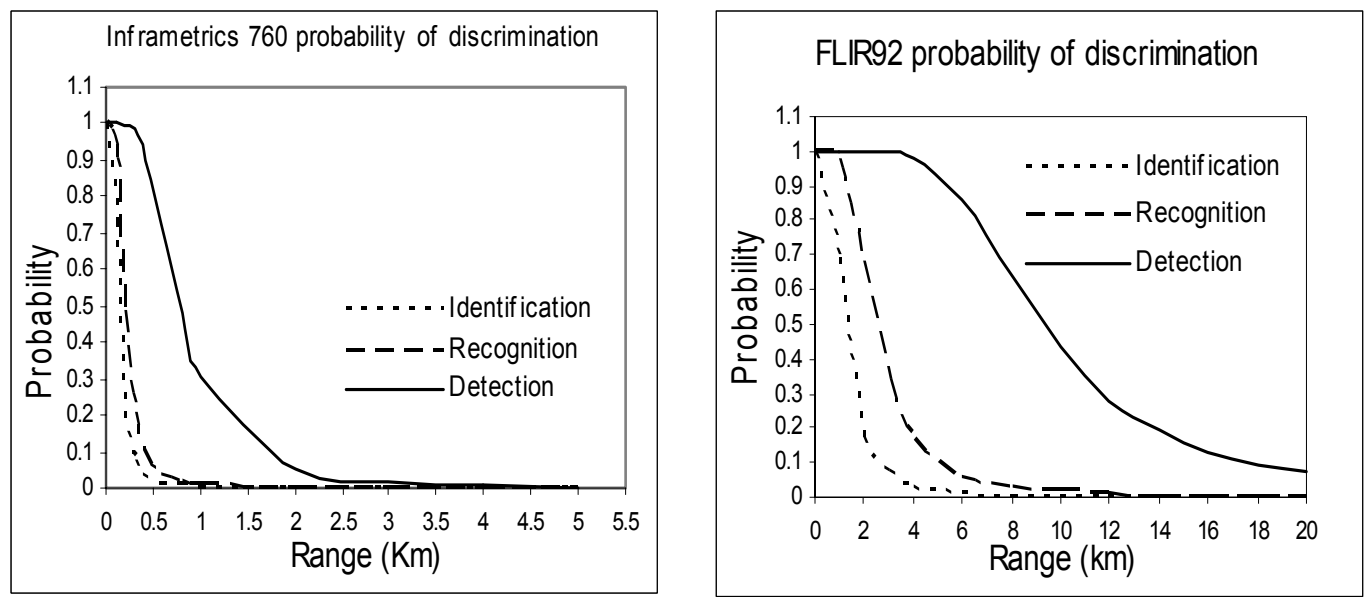

Fig. 3 illustrate the graphical representation of the $50 \%$ probabilities of identification, recognition and detection of the Inframetrics 760 and FLIR92. 
Fig.4 illustrate the target load line and the Inframetrics 760 and FLIR92 MRTD curves scaled for a detection range. The system MRTD curve is re-plotted with a detection range replacing the spatial frequency with a scaling factor equal 2.3 for Inframetrics 760 MRTD and a scaling factor equal 3.067 for FLIR92 MRTD. The apparent differential temperature of the target is plotted as a function of range. .It takes an $\mathrm{N}_{50}$ of 1.0 cycle across the target minimum dimension to obtain a $50 \%$ probability of detection for Inframetrics 760 TIR. The two lines cross at a point where the system provides the detection range $(0.79 \mathrm{~km})$ for a $50 \%$ probability of detection. It takes an $\mathrm{N}_{50}$ of 0.75 cycle across the target critical dimension to obtain a $50 \%$ probability of detection for FLIR92 TIR. The two lines cross at a point where the system provides the detection range $(9 \mathrm{~km})$ for a $50 \%$ probability of detection.
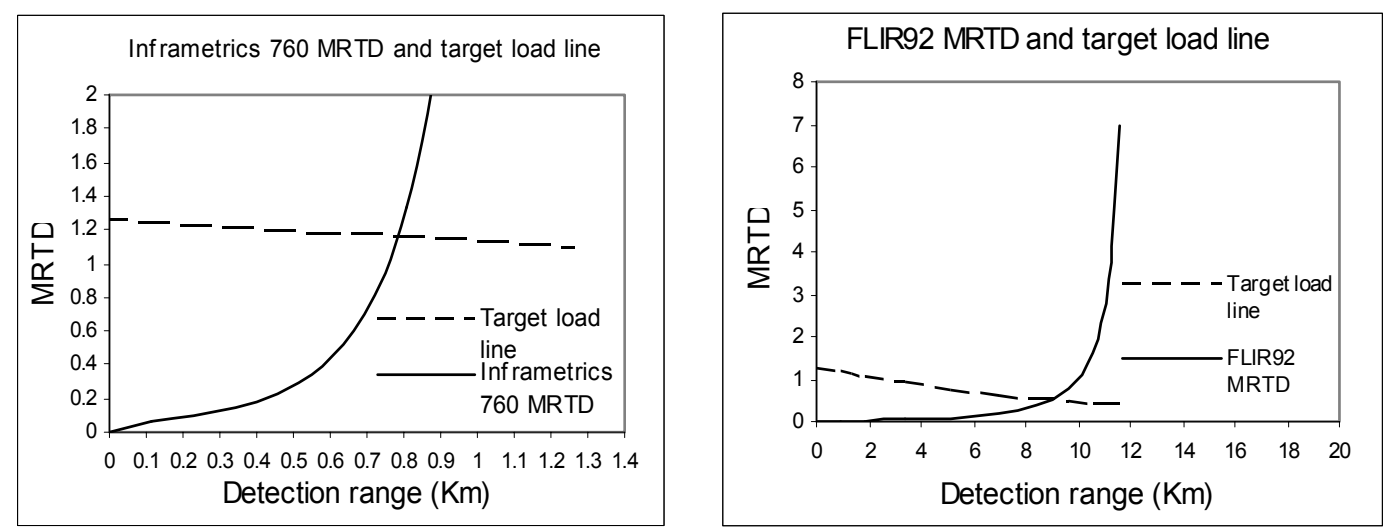

Fig.4 Inframetrics 760 and FLIR92 MRTD curves scaled for a detection range

Fig.5 illustrate the target load line and the Inframetrics 760 and FLIR92 MRTD curves scaled for a recognition range. The system MRTD curve is re-plotted with a recognition range replacing the spatial frequency with a scaling factor equal 0.575 for Inframetrics 760 MRTD and a scaling factor equal 0.767 for FLIR92 MRTD. The apparent differential temperature of the target is plotted as a function of range. .It takes an $\mathrm{N}_{50}$ of 4.0 cycle across the target minimum dimension to obtain a $50 \%$ probability of recognition for Inframetrics 760 TIR. The two lines cross at a point where the system provides the recognition range $(0.2 \mathrm{~km})$ for a $50 \%$ probability of recognition. It takes an $\mathrm{N}_{50}$ of 3.0 cycle across the target critical dimension to obtain a $50 \%$ probability of recognition for FLIR92 TIR. The two lines cross at a point where the system provides the recognition range $(2.5 \mathrm{~km})$ for a $50 \%$ probability of recognition.
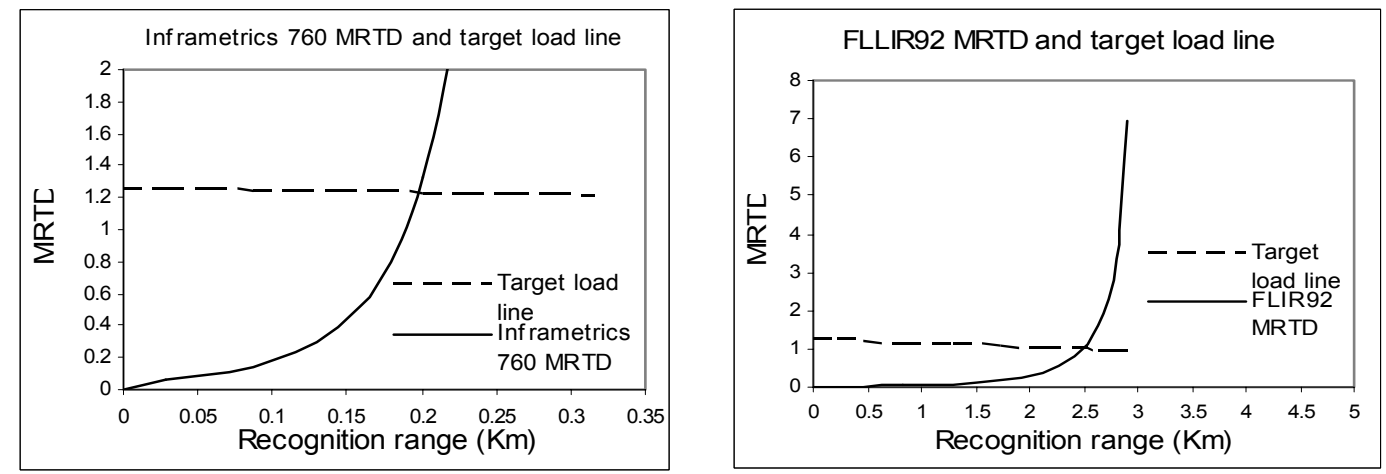

Fig.5 Inframetrics 760 and FLIR92 MRTD curves scaled for a recognition range 
Fig.6 illustrate the target load line and the Inframetrics 760 and FLIR92 MRTD curves scaled for a identification range. The system MRTD curve is re-plotted with a identification range replacing the spatial frequency with a scaling factor equal 0.2875 for Inframetrics 760 MRTD and a scaling factor equal 0.383 for FLIR92 MRTD. The apparent differential temperature of the target is plotted as a function of range. .It takes an $\mathrm{N}_{50}$ of 8.0 cycle across the target minimum dimension to obtain a $50 \%$ probability of identification for Inframetrics 760 TIR. The two lines cross at a point where the system provides the identification range $(0.1 \mathrm{~km})$ for a $50 \%$ probability of identification. It takes an $\mathrm{N}_{50}$ of 6.0 cycle across the target critical dimension to obtain a $50 \%$ probability of identification for FLIR92 TIR. The two lines cross at a point where the system provides the identification range $(1.25 \mathrm{~km})$ for a $50 \%$ probability of identification.
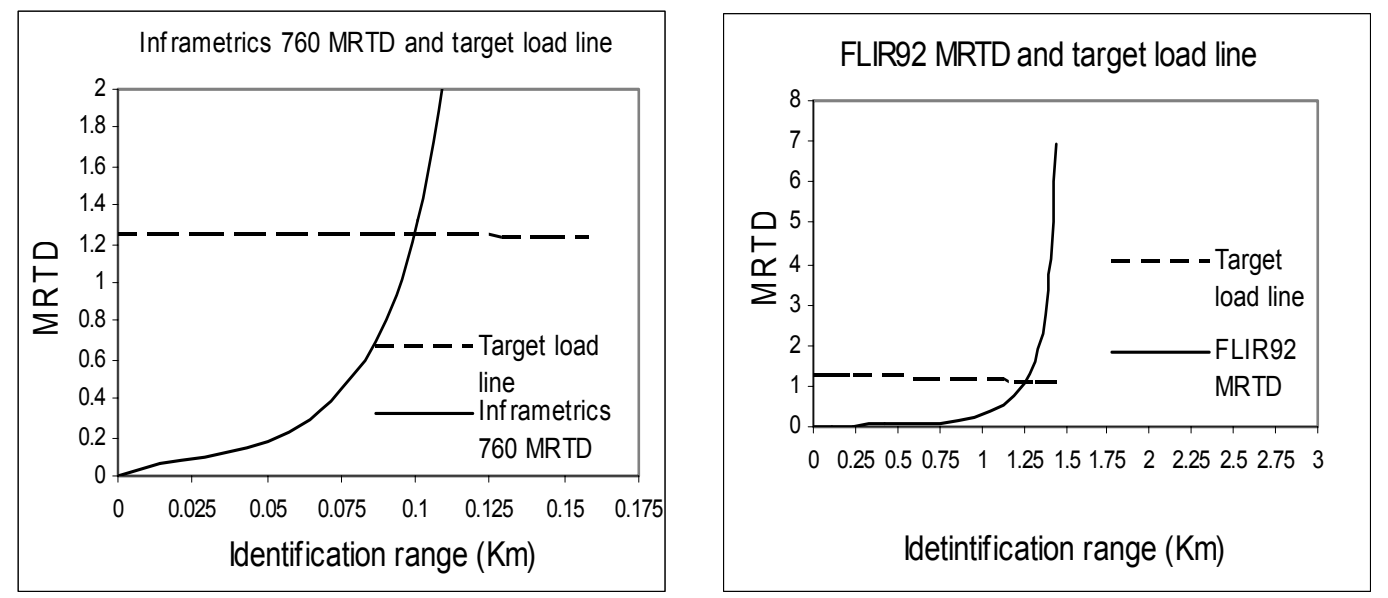

Fig.6 Inframetrics 760 and FLIR92 MRTD curves scaled for a identification range

\section{CONCLUSION}

In order to model the performance of a thermal imaging radiometer studying its behavior is needed. Obtaining the minimum resolvable temperature difference curve of the TIR enables that study. The results of the software program will allow the designer to determine the best MRTD curve of the TIR, which will give overall performance and hence probability of object discrimination and detection, recognition and identification ranges. This paper will facilitate and enhance the procedure that converse the TIR performance MRTD specification to an operation performance specification

\section{REFERENCES}

[1] G. Gassorgues " Infrared Thermogrphy ". Microwave technology series5.1998.

[2] Synery Network, "Inframetrics Model 760 Manual" .1992.

[3] C.W. Hoover and M.W. Curtis, "What in an MRTD?". SPIE, VOL.1488, PP 280-288,1993.

[4] Ronald G. Driggers " Introduction TO Infrared And Electro-optical Syetem".1999.

[5] "FLIR92 Thermal Imaging Systems Performance Model," U.S. Army Night Vision and Electronic Sensors Directorate, Ft. Belvoir (Virginia), Document 5008993, January 1993. 
[6] SPI Library, http://www.×20.org/library/thermal/infrared.html,2000.

[7] Boeing Flir, http:/www.boeing.com/defence-space/infoelect/flir,2000.

[8] Johnson, j., "Analysis of image forming radiometers," Image Intensifier Symposim, Warfare Vision Branch, Warfare Electrical Engineering Dept., U.S. Army Engineering Research and Development Laboratories, Ft. Belvoir, VA, 1958.

[9] Ratches, j., " Static Performance Model for Thermal Imaging Systems," Optical Engineering, Vol. 15, No.6, pp.252-530,1976. 